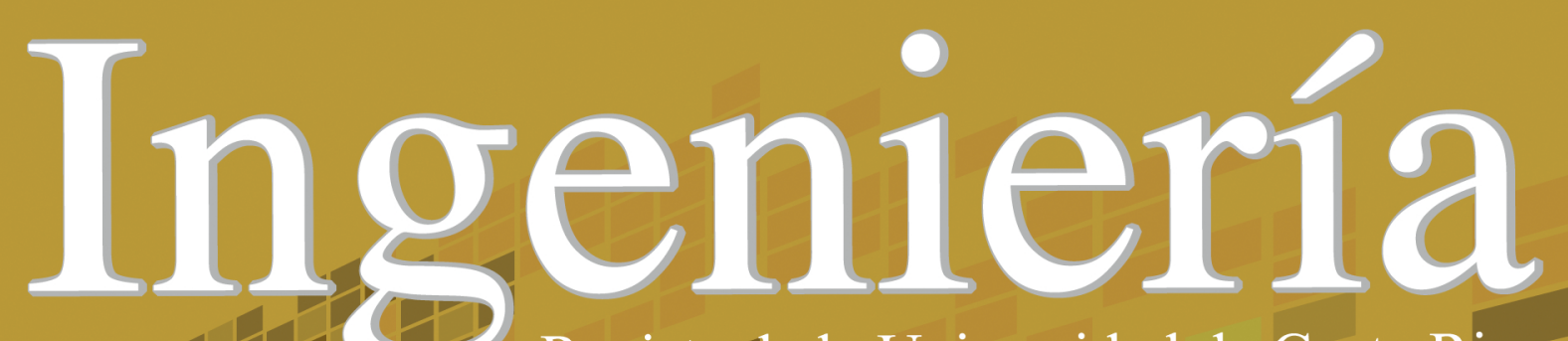

Revista de la Universidad de Costa Riea ENERO/JULIO 2013 - VOLUMEN 23 Número (1)

H

H

H71

Tr.

$x^{2}$

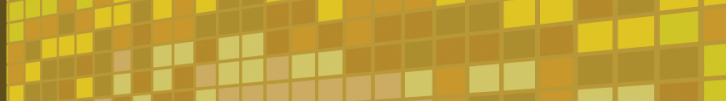

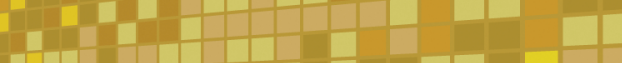

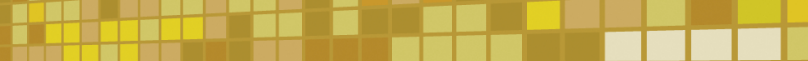

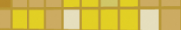

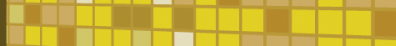

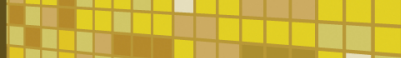

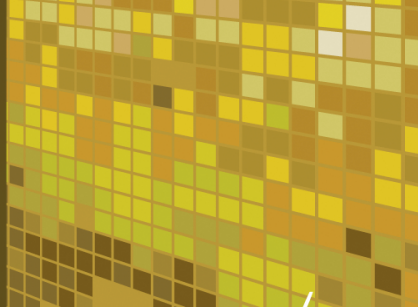

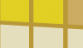



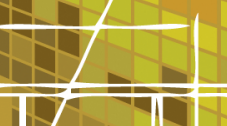

$+\infty \square$ 


\title{
ESTUDIO DE RETENCIÓN Y PENETRACIÓN DE TRES PRESERVANTES COMERCIALES EN NUEVE ESPECIES MADERABLES PRESENTES EN COSTA RICA
}

\author{
Johnny Daniel Alfaro Pérez
}

\begin{abstract}
Resumen
El objetivo de esta investigación fue determinar la retención y la penetración en nueve especies maderables presentes en Costa Rica, utilizando tres preservantes comerciales, de uso común en el país, con la finalidad de determinar el comportamiento de estas variables con respecto al tiempo de contacto, para muestras a nivel de laboratorio.

Para ello se prepararon muestras de $2 \mathrm{~cm}$ por $2 \mathrm{~cm}$ por $10 \mathrm{~cm}$ de cada una de las especies estudiadas, las cuales se preservaron por el método de inmersión-difusión, utilizando seis tiempos de contacto.

La retención fue determinada por la diferencia de masa entre la madera sin preservar y preservada, y la penetración se determinó utilizando reveladores químicos.

Se obtuvieron las curvas de retención contra tiempo para cada una de las muestras estudiadas, así como las curvas de penetración contra tiempo, y a partir de los resultados se determinó el tiempo para el cual al menos el $50 \%$ de muestras de cada especie alcanza ciertos valores de retención y penetración en probetas de tamaño laboratorio.
\end{abstract}

Palabras clave: Inmersión, Difusión, Preservantes, Tratamiento de preservación, Maderas tropicales

\begin{abstract}
The main goal of this research was to determine retention and penetration in nine woods present in Costa Rica, using three commercial wood preservatives, which are commonly use in the country, in order to determine the relationship between these variables and the contact time for laboratory size samples.

Samples of $2 \mathrm{~cm}$ by $2 \mathrm{~cm}$ by $10 \mathrm{~cm}$ each were prepared for each of the studied species, which were preserved using the immersion-diffusion method, using six contact times.

Retention was determined by the mass difference between treated and not treated wood, and penetration was determined using changes of color by chemical reactions.

For each of the studied species, it was obtained retention versus time graphs and penetration versus time graphs. From these results it was determined the contact time in which at least half of the samples of each species reach retention and penetration fix values for lab-size samples.
\end{abstract}

Keywords: Immersion, Diffusion, Wood Preservatives, Preservation treatment, Tropical Hardwoods.

Recibido: 11 de marzo del 2013 • Aprobado: 11 de junio del 2013

\section{INTRODUCCIÓN}

La madera es un material polimérico natural, que anatómicamente corresponde al xilema secundario de las plantas leñosas, producto del proceso de división celular del cambium vascular (Mora y Encinas, 2006).
Al ser un material biológico, el mismo se encuentra propenso al ataque de ciertos agentes físicos, químicos y biológicos en detrimento de sus características físicas, químicas, mecánicas y de durabilidad (Flores, 1999).

Entre los agentes deterioradores de la madera se encuentran los agentes abióticos 
como "weathering" o fotodegradación por luz ultravioleta, descomposición térmica y química, así como desgaste mecánico. Por su parte, los agentes deterioradores bióticos de la madera son hongos y bacterias, así como insectos xilófagos y barrenadores marinos (Lepage, et al, 1986).

Existen dos tipos de medidas que se pueden tomar para controlar el biodeterioro de la madera: preventivo o curativo. Las medidas preventivas pueden ser la disminución de la humedad de la madera, aplicación de preservantes o tratamiento con químicos en el lugar de aplicación (IPT, 2001).

El principio por el cual funcionan los preservantes químicos es porque son tóxicos a los organismos biológicos que atacan la madera. Los ingredientes activos en las distintas formulaciones son varios y cada uno posee un modo de acción distinto, aunque algunos todavía son desconocidos (Ibach, 2005).

Los preservantes se pueden agrupar en tres categorías, de acuerdo a sus características químicas y de aplicación: (IPT, 2001)

- Oleosos: productos esencialmente representados por los derivados del alquitrán de hulla.

- Oleosolubles: productos que contienen mezclas complejas de fungicidas $\mathrm{y} / \mathrm{o}$ insecticidas, donde los compuestos son de naturaleza orgánica u organometálica. Por ejemplo: creosota, pentaclorofenol, naftalenato de cobre, TBTO.

- Hidrosolubles: productos que tienen mezclas más o menos complejas de sales metálicas. Por ejemplo: CCA, ACZA, CAC, ACC, ACA, CZC, CCB.

Se ha investigado el uso de nuevos preservantes, más amigables con el ambiente y con la salud humana como el Wolmanit CX-8, Tanalith E (Yildiz, Temiz, Derya, Yildiz, 2004), MCQ, DDAC (Zelinka, Sidner y Stone, 2010), compuestos de cobre-etanolamina (Humar y Lesar, 2008) y otros más, en sustitución de algunos preservantes que ya no pueden ser utilizados a nivel comercial como el pentaclorofenol, la creosota y el CCA.

Sin embargo, muchos de estos preservantes cuentan con marcas comerciales y no se distribuyen actualmente en el país; por ello, es de suma importancia poder estudiar aquellos que sí se encuentran accesibles a nivel comercial.

Los procesos más utilizados para la aplicación de preservantes, a nivel comercial, se pueden clasificar en dos: de corta y larga duración de protección. Para el caso de la protección de larga duración, que es de interés en este proyecto, se puede subclasificar en aplicación del preservante con y sin presión (Zabel y Morrell, 1992).

Para el caso de los procesos de aplicación del preservante sin presión, se presentan los siguientes: (IPT, 2001)

- Aspersión: se rocía el preservante sobre la madera por medio de aspersores o pulverizadores que promueve un flujo continúo del químico a través de la madera.

- Pincelado: proceso simple, donde por medio de una brocha se distribuye el preservante. Tiene un bajo rendimiento.

- Inmersión: se sumerge la madera en un tanque que contiene el preservante en un periodo variable.

En el proceso por inmersión para garantizar la absorción del preservante y garantizar la efectividad del tratamiento se debe sumergir la madera el tiempo necesario, que puede variar de unos cuantos minutos a horas, dependiendo del uso final (AWPA, 2012).

Se conoce que la penetración y la absorción del preservante en los distintos cortes de la madera son diferentes, y que la capacidad de absorción y penetración se encuentran en función del tiempo, donde se llega asintóticamente a un valor determinado (Purslow, 1976).

Los procesos sin presión presentan la desventaja que la penetración del preservante y su distribución es altamente variable, así como el límite tiempo de protección comparado con los métodos a presión. Sin embargo, los métodos a presión requieren de equipos especiales y de alto costo (Zabel y Morrell, 1992).

En cuanto al estudio de las especies maderables de Costa Rica, se han realizado estudios de preservación a presión-vacío (Blanco y Cruz, 1999; Blanco, Carpio, Cruz, Govaere; 2008); sin embargo, los tiempos de inmersión sólo se han estudiado para melina de la zona Atlántica con sales de boro (Moya, 
Leandro, Monge, 2004; Berrocal, Muñoz, González, 2004).

En nuestro país, el principal problema del uso de los métodos presión-vacío es su costo y el bajo número de aserraderos o plantas de preservación que cuentan con el equipo necesario para hacer este trabajo; lo que hace del método a presión poco práctico para el sector en general; por ello, lo más frecuente y sencillo es el uso de preservación por inmersión o pintado, de ahí la importancia de conocer la efectividad de estos tratamientos, así como el tiempo más adecuado.

Por lo tanto, en esta investigación se plantea como objetivo evaluar la penetración de tres preservantes asequibles a nivel comercial en la madera, por el método de preservación por inmersión, a partir de muestras de nueve especies maderables, expuestas a distintos tiempos de contacto, de manera que los resultados obtenidos permiten cuantificar la retención y la penetración del preservante y se puede obtener un tiempo adecuado de contacto.

\section{METODOLOGÍA EXPERIMENTAL}

\subsection{Variables}

\section{Variables de estudio:}

Son aquellas que son de interés para el trabajo y que responden al objetivo general planteado. De esta manera, se tienen las siguientes:

- Penetración: dado en mm. Representa la distancia que el preservante logra atravesar a lo largo de la madera en un tiempo dado.

- Retención: dado en $\mathrm{kg} / \mathrm{m} 3$. Representa la cantidad de preservante absorbido por la madera en relación con su volumen para un tiempo dado.

\section{Variables independientes:}

Son aquellas variables que se alteran de forma arbitraria para determinar su efecto sobre las variables de respuesta. En concordancia con esta definición, se presentan las siguientes:

- Especie maderable: cada especie tiene un comportamiento distinto durante el proceso de inmersión, debido a sus características morfológicas y anatómicas. En el Cuadro 1 se muestran los nombres científicos de las especies estudiadas.

- Preservante: se eligen preservantes comerciales, disponibles en Costa Rica, que tienen principios activos distintos. En el Cuadro 2 se muestra la codificación, así como sus principios activos y composiciones brindadas por el respectivo fabricante. Los preservantes fueron preparados a partir de la formulación concentrada de fábrica y diluidos según las especificaciones del fabricante. El preservante código 3 no se diluye en agua por instrucciones del fabricante.

\section{Tiempo de preservación:}

Se eligen distintos tiempos para la aplicación del preservante: $0,1,6,24,48$ y 72 horas. Empíricamente se sabe que a tiempos de inmersión muy cortos, la difusión del preservante en la madera es muy lenta, por lo que, la penetración es pequeña y muchas veces insuficiente, dependiendo del tipo de aplicación final de la madera tratada. Por otro lado, a tiempos muy largos se puede saturar la madera, por lo que, ya no absorbe más preservante, o la penetración presentada es mucho mayor a la aconsejada, generando un gasto innecesario de recursos y tiempo para las empresas.

\section{Variables fijas:}

Son aquellas que se mantienen sin modificar a lo largo de la experimentación, con valores arbitrarios. En este caso, las variables fijas fueron las dimensiones de las muestras, que se definieron de 2 $\mathrm{cm}$ de ancho por $2 \mathrm{~cm}$ de alto por $10 \mathrm{~cm}$ de largo.

Variables de respuesta:

Son aquellas que se modifican por la acción de las variables independientes. En este caso, las 
Cuadro 1. Niveles de las especies maderables

\begin{tabular}{|c|c|c|c|}
\hline Código & Especie & Nombre Común & Familia \\
\hline GP & Hymenaea courbaril (L.) & Guapinol & Caesalpiniaceae \\
\hline $\mathrm{RC}$ & Terminalia amazonia (S.F.Gmel.) Exell. & $\begin{array}{l}\text { Roble coral o } \\
\text { amarillón }\end{array}$ & Combretaceae \\
\hline PI & Hieronyma alchorneoides (Fr. Allem.) & Pilón & Euphorbiaceae \\
\hline $\mathrm{CD}$ & Cedrela odorata (L.) & Cedro amargo & Meliaceae \\
\hline $\mathrm{TE}$ & Tectona grandis (L.f.) & Teca & Verbenaceae \\
\hline $\mathrm{CI}$ & Cupressus lusitanica (Mill.) & Ciprés & Cupressaceae \\
\hline GN & Enterolobium cyclocarpum (Jacq.) Gris. & Guanacaste & Mimosaceae \\
\hline $\mathrm{CM}$ & Calophyllum brasiliense (Camb.) & Cedro María & Clusiaceae \\
\hline ME & Gmelina arborea (Roxb.) & Melina & Verbenaceae \\
\hline
\end{tabular}

Fuente: Elaboración propia, 2013.

Cuadro 2. Niveles de los tipos de preservantes

\begin{tabular}{ccc}
\hline Código & Principio activo & $\begin{array}{c}\text { Composición química del } \\
\text { principio activo concentrado }\end{array}$ \\
\hline 1 & Boro & $49 \%$ Óxido de boro \\
2 & Cromo-cobre-boro & $\begin{array}{c}2 \% \text { óxido de cromo, } 5 \% \text { óxido de } \\
\text { boro y } 25 \% \text { de óxido de cobre }\end{array}$ \\
3 & Zinc & Naftalenato de zinc* \\
\hline
\end{tabular}

*Composición no proporcionada por el fabricante. 
variables de respuesta son las mismas que las variables de estudio: la penetración y la retención.

\subsection{Diseño experimental}

Se aplica a cada especie maderable un tipo específico de preservante, para cada uno de los tiempos previamente definidos. Además, se preparan 10 réplicas para cada una de las combinaciones posibles con la finalidad de incrementar la confiabilidad de los resultados obtenidos, dando un total de muestras de 180 por especie, para un total de 1620.

\subsection{Preparación del material}

Las muestras fueron lijadas, se rotularon, se colocaron en un horno de convección por 24 horas, luego, se dejaron enfriar en un desecador y se determinó la masa de cada una de las muestras. Las muestras se mantuvieron en un cuarto de condiciones controladas de 22 ${ }^{\circ} \mathrm{C}$ y $60 \%$ de humedad relativa hasta que las muestras se estabilizaron para tener un $12 \%$ de humedad. Las probetas fueron elaboradas para distinguir claramente la dirección transversal y la longitudinal de la madera.

\subsection{Preservación de las muestras}

Para la preservación de las muestras se utilizaron jaulas construidas con alambre, de manera que se mantengan las muestras en su lugar durante la preservación y evitar que las mismas floten. A las muestras se le midieron sus dimensiones utilizando un Vernier digital, así como sus masas iniciales (a un $12 \%$ de contenido de humedad) utilizando una balanza. Posteriormente, cada muestra se coloca en su respectiva jaula y se deja por el tiempo determinado de tratamiento.

Una vez terminado el tiempo de tratamiento, las muestras se sacan de las jaulas y se dejan secar al aire por un período mínimo de 24 horas, antes de pasar al cuarto de acondicionamiento con las mismas condiciones controladas para regresar a un $12 \%$ de contenido de humedad.

\subsection{Preparación de reveladores}

Debido a que el principio activo de cada uno de los preservantes utilizados es distinto, se deben elaborar tres reveladores químicos distintos. Para ello, se utilizan las normas AWPA-A3-08 (para los reveladores de boro y cromo), y AWPA-A3-91 (para el revelador de zinc).

\subsection{Determinación de retención y penetración}

Una vez que las muestras se estabilizan en el cuarto de condiciones controladas (12\% de humedad), se determina su nueva masa y se calcula la retención del preservante para cada una de las muestras utilizando la siguiente ecuación:

$$
\frac{\mathrm{m}_{\mathrm{f}}-\mathrm{m}_{\mathrm{i}}}{V}
$$

Donde:

mi es la masa inicial (a $12 \%$ humedad) en $\mathrm{kg}$. $m f \quad$ es la masa final (a $12 \%$ humedad) en $\mathrm{kg}$. $V \quad$ es el volumen de la muestra en $\mathrm{m} 3$.

Cada muestra se corta de forma transversal y longitudinalmente, y se aplica el respectivo revelador. Utilizando un Vernier digital se mide la penetración del preservante para cada una de las muestras, reportándose el promedio de dos medidas.

\section{ANÁLISIS DE RESULTADOS}

Se obtuvieron las curvas de retención y penetración para cada combinación de preservante y especie, tanto transversal como lateral (de acuerdo a las direcciones en que se pudo elaborar las muestras), de manera que se puede apreciar la variación con el tiempo de inmersión de estas variables. Sin embargo, debido a la gran cantidad de gráficos, se presentan a manera de ejemplo las Figuras 1 a 3, 


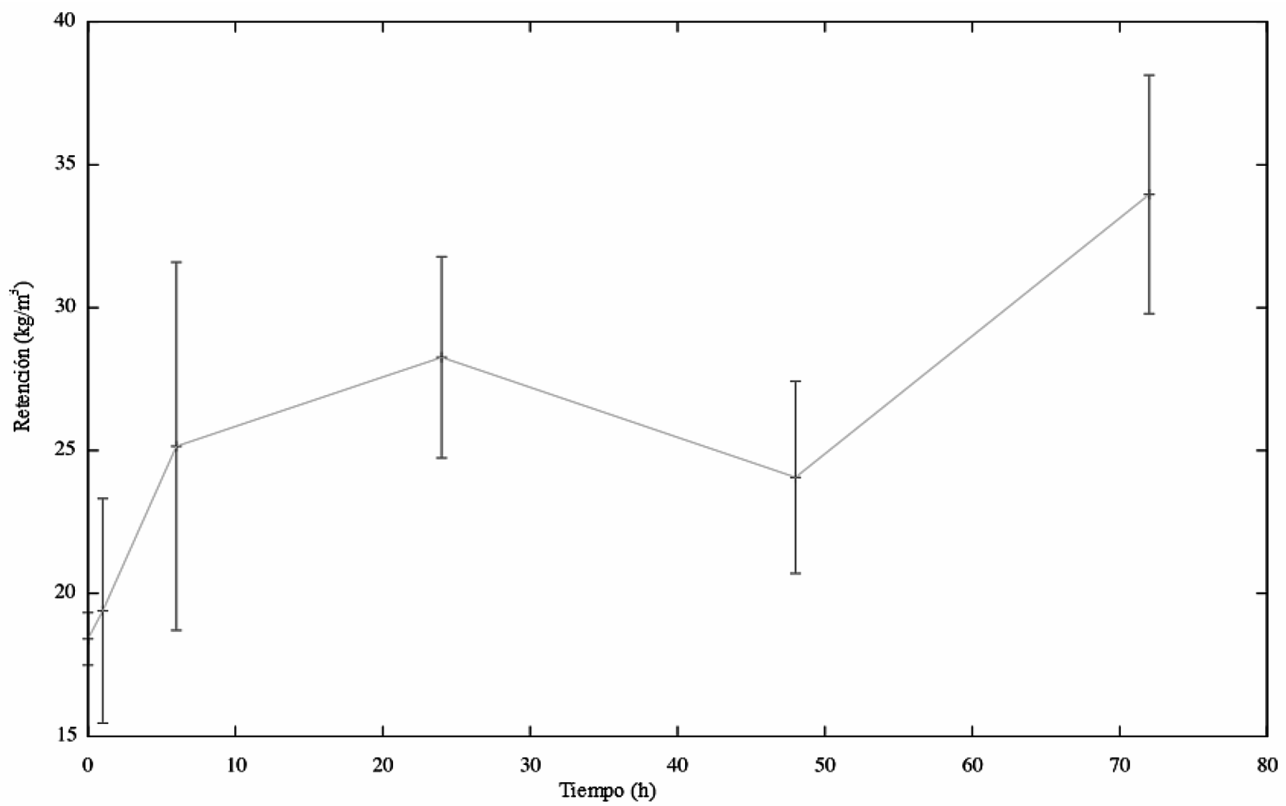

Figura 1. Curva de retención contra tiempo para H. courbaril.

Fuente: Elaboración propia, 2013.

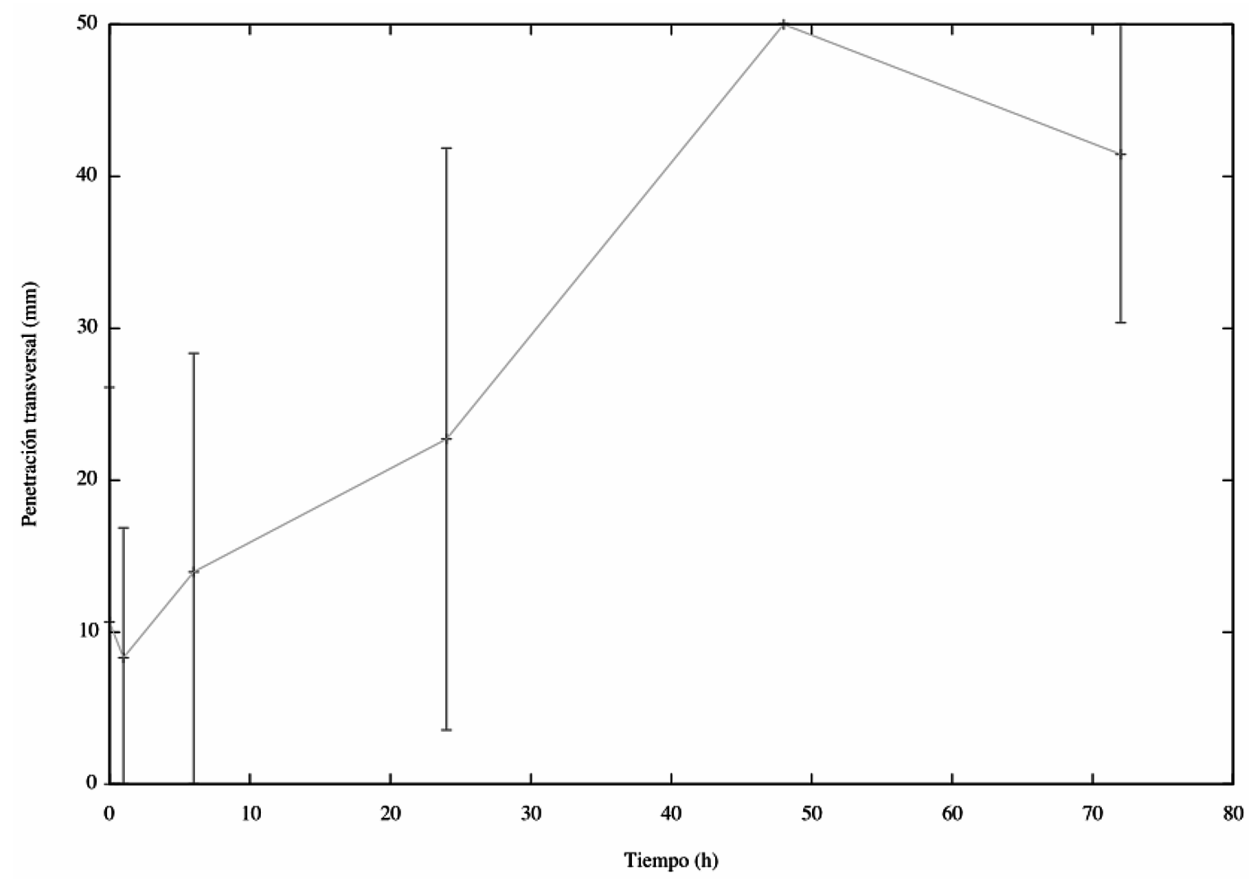

Figura 2. Curva de penetración transversal contra tiempo para H. courbaril. Fuente: Elaboración propia, 2013. 


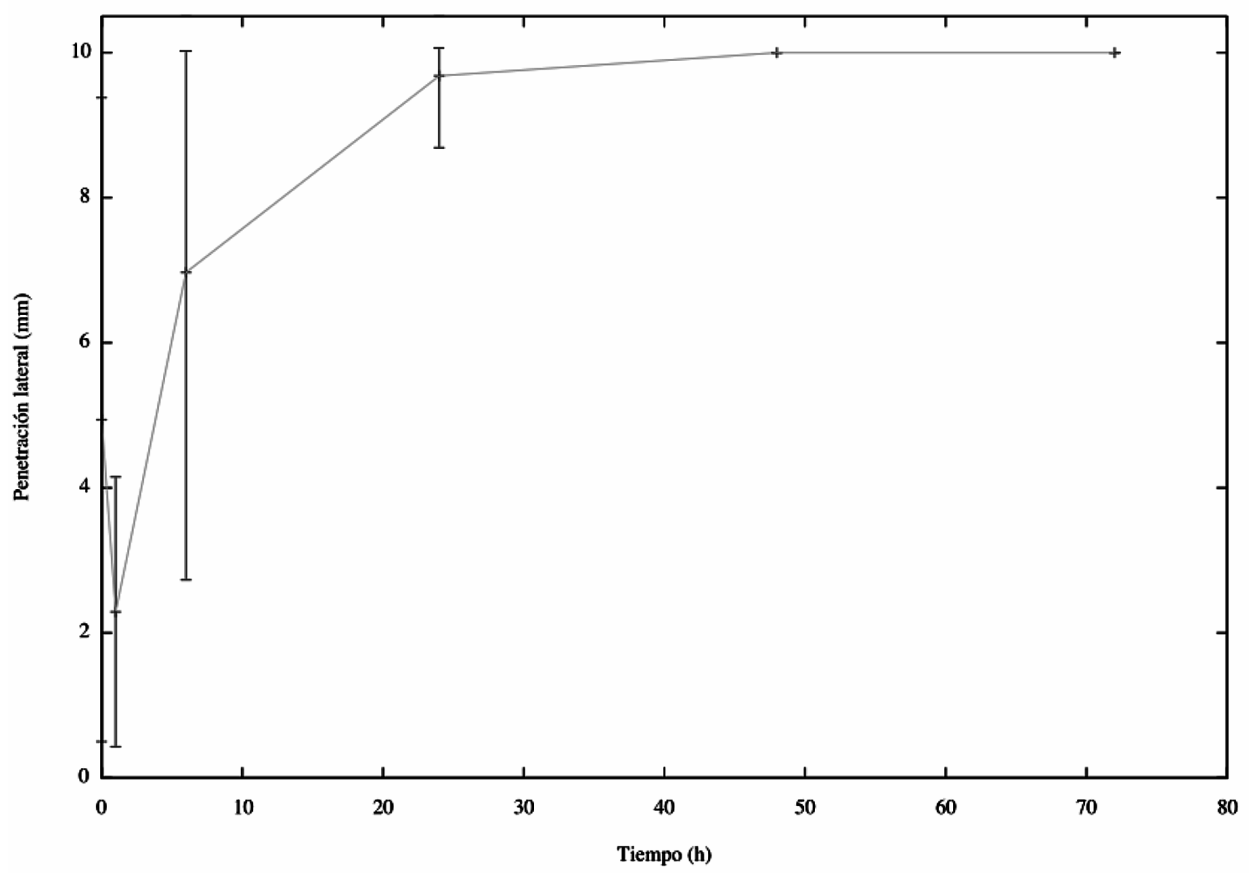

Figura 3. Curva de penetración lateral contra tiempo para H. courbaril.

Fuente: Elaboración propia, 2013.

donde se ilustra el caso de H. courbaril tratado con el preservante código 1. Las demás figuras se pueden consultar en Alfaro (2013).

Para el análisis del tratamiento aplicado en cada especie se toma en cuenta que en las normas internacionales existentes (como la norma AWPA U1-12), no se regulan la penetración y la retención de las especies estudiadas en este proyecto, para ninguna aplicación en especial, ni con los preservantes utilizados.

Por ello, se va utilizar un valor fijo para cada parámetro estudiado, el cual está basado en las siguientes observaciones: a menor valor se corre el riesgo de que el preservante no sea eficaz en la preservación de la madera, y a un valor mayor la protección brindada es más de la necesaria, resultando en un desperdicio del preservante.

Además, la obtención del valor fijo (Cuadros 3 a 5) se toma de acuerdo al valor del percentil 50 (es decir, cuando se ordenan todos los valores de cada muestra estudiada por especie, se obtiene el valor de penetración o retención de la medida central, lo que implica que el $50 \%$ de las muestras tienen un valor mayor o menor al presentado) obtenido para cada parámetro en estudio, para cada especie y tipo de preservante aplicado.

En los Cuadros 3 al 5 se determina el valor del percentil 50, tanto de retención y penetración por especie y tipo de preservante, así como el tiempo mínimo necesario para que se alcancen dichos valores.

En el Cuadro 6 se estima cada dato considerando el valor del percentil 50 de cada especie clasificada (según sea fácil o difícil relativamente de preservar) y se realiza un promedio. Además, la facilidad relativa de preservación (cuya clasificación por especie) se muestra en el Cuadro 7.

Del Cuadro 6 se puede observar que para especies fáciles de preservar, los valores promedios de retención son menores; sin embargo, para determinar la efectividad del tratamiento, es decir, si la retención obtenida promedio es adecuada, son necesarios estudios de deterioro biológico para las condiciones obtenidas. 
Cuadro 3. Tiempo observado para preservación de madera con preservante código 1, por variable

\begin{tabular}{|c|c|c|c|c|c|c|}
\hline $\begin{array}{l}\text { Nombre } \\
\text { científico }\end{array}$ & $\begin{array}{l}\text { Retención } \\
\qquad\left(\mathbf{k g} / \mathbf{m}^{3}\right)\end{array}$ & $\begin{array}{l}\text { Tiempo } \\
\text { (h) }\end{array}$ & $\begin{array}{c}\text { Penetración } \\
\text { transversal } \\
(\mathbf{m m})\end{array}$ & $\begin{array}{c}\text { Tiempo } \\
\text { (h) }\end{array}$ & $\begin{array}{c}\text { Penetración } \\
\text { lateral } \\
(\mathbf{m m})\end{array}$ & $\begin{array}{c}\text { Tiempo } \\
\text { (h) }\end{array}$ \\
\hline H. courbaril & 23,2 & 5 & 16,3 & 12,5 & 10,0 & 48 \\
\hline T. amazonia & 12,9 & 3,5 & 13,2 & 1 & 5,2 & 1 \\
\hline $\begin{array}{c}H . \\
\text { alchorneoides }\end{array}$ & 8,1 & 1 & 10,0 & 1 & 1,8 & 12 \\
\hline C. odorata & 5,7 & 1 & 24,3 & 1 & 6,8 & 24 \\
\hline T. grandis & 9,1 & 1 & 7,0 & 1 & 2,3 & 1 \\
\hline C. lusitanica & 14,9 & 8 & 2,6 & 1 & 3,6 & 1 \\
\hline E. cyclocarpum & 7,0 & 2,5 & 25,2 & 14 & 10,0 & 48 \\
\hline C. brasiliense & 15,0 & 17 & 6,4 & 1 & 3,1 & 12 \\
\hline G. arborea & 8,7 & 1 & 20,5 & 4 & 6,4 & 1 \\
\hline
\end{tabular}

Fuente: Elaboración propia, 2013.

Cuadro 4. Tiempo observado para preservación de madera con preservante código 2, por variable

\begin{tabular}{|c|c|c|c|c|c|c|}
\hline $\begin{array}{l}\text { Nombre } \\
\text { científico }\end{array}$ & $\begin{array}{l}\text { Retención } \\
\left(\mathbf{k g} / \mathbf{m}^{3}\right)\end{array}$ & $\begin{array}{c}\text { Tiempo } \\
\text { (h) }\end{array}$ & $\begin{array}{c}\text { Penetración } \\
\text { transversal } \\
(\mathrm{mm})\end{array}$ & $\begin{array}{c}\text { Tiempo } \\
\text { (h) }\end{array}$ & $\begin{array}{c}\text { Penetración } \\
\text { lateral } \\
(\mathbf{m m})\end{array}$ & $\begin{array}{c}\text { Tiempo } \\
\text { (h) }\end{array}$ \\
\hline H. courbaril & 23,2 & 4,5 & 5,3 & 7 & 10,0 & 4 \\
\hline T. amazonia & 12,9 & 10 & 2,6 & 7,5 & 5,2 & 1 \\
\hline $\begin{array}{c}H . \\
\text { alchorneoides }\end{array}$ & 8,7 & 1 & 7,1 & 1 & 1,8 & 8 \\
\hline C. odorata & 4,4 & 1 & 10,6 & 1 & 6,8 & 1 \\
\hline T. grandis & 9,7 & 1 & 2,3 & 11 & 2,3 & 1 \\
\hline C. lusitanica & 14,9 & 16 & 2,1 & 3 & 3,6 & 10 \\
\hline E. cyclocarpum & 7,5 & 4 & 6,4 & 6 & 10,0 & 12 \\
\hline C. brasiliense & 45,6 & 6 & 4,4 & 10 & 3,1 & 1 \\
\hline G. arborea & 8,7 & 1 & 5,1 & 1 & 3,6 & 5 \\
\hline
\end{tabular}


Cuadro 5. Tiempo observado para preservación de madera con preservante código 1, por variable

\begin{tabular}{|c|c|c|c|c|c|c|}
\hline $\begin{array}{l}\text { Nombre } \\
\text { científico }\end{array}$ & $\begin{array}{c}\text { Retención } \\
\left(\mathbf{k g} / \mathbf{m}^{\mathbf{3}}\right)\end{array}$ & $\begin{array}{c}\text { Tiempo } \\
\text { (h) }\end{array}$ & $\begin{array}{c}\text { Penetración } \\
\text { transversal } \\
(\mathrm{mm})\end{array}$ & $\begin{array}{c}\text { Tiempo } \\
\text { (h) }\end{array}$ & $\begin{array}{c}\text { Penetración } \\
\text { lateral } \\
(\mathbf{m m})\end{array}$ & $\begin{array}{c}\text { Tiempo } \\
\text { (h) }\end{array}$ \\
\hline H. courbaril & 16,1 & 3 & 50,0 & 1 & 10,0 & 1 \\
\hline T. amazonia & 6,5 & 10 & 6,5 & 12 & 10,0 & 48 \\
\hline $\begin{array}{c}H . \\
\text { alchorneoides }\end{array}$ & 13,1 & 1 & 50,0 & 24 & 10,0 & 1 \\
\hline C.odorata & 4,0 & 18 & 50,0 & 6 & 10,0 & 1 \\
\hline T. grandis & 1,4 & 1 & 2,6 & 1 & 4,0 & 1 \\
\hline C. lusitanica & 7,5 & 4 & 7,3 & 1 & 6,3 & 1 \\
\hline E. cyclocarpum & 8,0 & 12 & 50,0 & 1 & 10,0 & 1 \\
\hline C. brasiliense & 9,9 & 8 & 50,0 & 6 & 10,0 & 1 \\
\hline G. arborea & 4,1 & 1 & 2,5 & 1 & 3,8 & 1 \\
\hline
\end{tabular}

Fuente: Elaboración propia, 2013.

Cuadro 6. Valores promedio del percentil 50 de retención y penetración por tipo de preservante

$\left.\begin{array}{ccccc}\hline \text { Preservante } & \begin{array}{c}\text { Facilidad de } \\ \text { preservación }\end{array} & \begin{array}{c}\text { Retención } \\ \left(\mathbf{k g} / \mathbf{m}^{\mathbf{3}}\right)\end{array} & \begin{array}{c}\text { Penetración } \\ \text { transversal } \\ (\mathbf{m m})\end{array} & \begin{array}{c}\text { Penetración } \\ \text { lateral }\end{array} \\ (\mathbf{m m})\end{array}\right)$

Fuente: Elaboración propia, 2013. 
Cuadro 7. Facilidad relativa de presentación de las especies

\begin{tabular}{ccc}
\hline Nombre científico & Peso específico & Preservación \\
H. courbaril & 0,79 & Difícil \\
\hline T. amazonia & 0,68 & Difícil \\
H. alchorneoides & 0,63 & Fácil \\
C. odorata & 0,43 & Fácil \\
T. grandis & 0,61 & Duramen: muy difícil \\
C. lusitanica & 0,43 & Albura: fácil \\
E. cyclocarpum & 0,35 & Moderadamente fácil \\
C. brasiliense & 0,47 & Fácil \\
G. arborea & 0,34 & Difícil \\
\hline
\end{tabular}

Fuente: Carpio, 1992.

En cuanto a la penetración, debido a que cada preservante es hecho a base de solventes distintos y que la solubilidad del principio activo es distinta, los valores no muestran ninguna tendencia entre especies fáciles y difíciles de preservar; inclusive, no se encuentran tendencias cuando se analizan estos mismos datos de los Cuadros 3 a 5 .

Se puede observar que para cada especie, el tiempo mínimo para uno de los valores (retención o penetración) puede ser inferior al tiempo mínimo necesario para satisfacer los otros.

Por ejemplo, en el caso de la especie $H$. courbaril, se tiene que el valor de retención se satisface (por interpolación de la Figura 1) a las 5 horas; sin embargo, la penetración transversal (según la Figura 2) en ese tiempo es de apenas 13 $\mathrm{mm}$, y la penetración lateral (según la Figura 3) es de apenas $6 \mathrm{~mm}$. Por lo tanto, si lo que se quiere es obtener un tiempo en el que las tres variables alcancen los valores del Cuadro 3, entonces, se debería escoger el tiempo mayor.

Con esto se asegura que al menos el $50 \%$ de las piezas que se preserven en ese tiempo, tienen una alta probabilidad de presentar los valores de retención y penetración (tanto transversal como lateral) descritos. Por supuesto, un mayor tiempo de inmersión se puede realizar; pero, se debe analizar en cada caso particular si se obtiene algún beneficio económico en ello. Esta comprobación se sale del objetivo del presente trabajo. Dichos tiempos de inmersión se pueden determinar gráficamente utilizando las figuras (para un total de 81 figuras distintas) que se presentan en Alfaro (2013).

En los Cuadros 4 y 5 se presentan los mismos valores, en este caso para los preservantes código 2 y 3 , respectivamente, y en el Cuadro 8 se presenta el tiempo requerido para que al menos el $50 \%$ de las muestras alcancen todos los valores de retención y penetración presentados en los Cuadros 3 a 5. Por lo tanto, estos tiempos pueden servir como un marco de referencia para el uso de este tipo de tratamiento en las especies estudiadas.

Es importante tomar en cuenta que la experimentación se realiza en probetas de tamaño laboratorio, de manera que, si los resultados desean 
Cuadro 8. Tiempo observado para alcanzar todos los parámetros estudiados por especie

\begin{tabular}{|c|c|c|c|c|c|c|}
\hline $\begin{array}{l}\text { Nombre } \\
\text { científico }\end{array}$ & $\begin{array}{c}\text { Código } \\
\text { preservante }\end{array}$ & $\begin{array}{c}\text { Tiempo } \\
\text { (h) }\end{array}$ & $\begin{array}{c}\text { Código } \\
\text { preservante }\end{array}$ & $\begin{array}{c}\text { Tiempo } \\
\text { (h) }\end{array}$ & $\begin{array}{c}\text { Código } \\
\text { preservante }\end{array}$ & $\begin{array}{c}\text { Tiempo } \\
\text { (h) }\end{array}$ \\
\hline H. courbaril & 1 & 48 & 2 & 7 & 3 & 3 \\
\hline T. amazonia & 1 & 3,5 & 2 & 10 & 3 & 48 \\
\hline $\begin{array}{c}H . \\
\text { alchorneoides }\end{array}$ & 1 & 12 & 2 & 8 & 3 & 24 \\
\hline C. odorata & 1 & 24 & 2 & 1 & 3 & 18 \\
\hline T. grandis & 1 & 1 & 2 & 11 & 3 & 1 \\
\hline C. lusitanica & 1 & 8 & 2 & 16 & 3 & 4 \\
\hline E. cyclocarpum & 1 & 48 & 2 & 12 & 3 & 12 \\
\hline C. brasiliense & 1 & 17 & 2 & 10 & 3 & 8 \\
\hline G. arborea & 1 & 4 & 2 & 5 & 3 & 1 \\
\hline
\end{tabular}

Fuente: Elaboración propia, 2013.

ser aplicados a nivel industrial, se debe realizar un experimento que demuestre el cambio de escala; por lo tanto, los resultados presentados en este trabajo sirven como un marco de referencia para futuras experimentaciones que vayan a realizar los usuarios finales que se encuentren interesados en realizarlas.

En lo concerniente a la tendencia que se presentan en las variables de penetración y retención contra el tiempo, para la mayor cantidad de combinaciones entre especies y preservante, no se encuentra una relación funcional asintótica, que era la funcionalidad esperada según la literatura consultada (Purslow, 1976).

La presencia de máximos (como en el caso de las Figuras 1 a 3) y mínimos (como en el caso de la Figura 1) se debe a características anatómicas propias de cada especie maderable, así como de la naturaleza del preservante. Por ejemplo, en el caso del preservante código 1, el disolvente es agua y el agente activo son sales de boro, las cuales se difunden a través de la estructura de la madera, y los cristales de la sal se adhieren a las paredes de los elementos anatómicos; por tanto, puede ocurrir una saturación de los cristales en cierto momento, impidiendo una mayor retención del presente en la madera.

Un estudio más profundo acerca de los lugares donde ocurren estas saturaciones en la estructura de la madera se podría realizar para cada especie; sin embargo, esto se sale de los objetivos de la presente investigación.

\section{CONCLUSIONES}

- Se lograron preservar las muestras de nueve especies maderables presentes en Costa Rica, utilizando el método de inmersióndifusión, en seis tiempos distintos, con tres preservantes de uso comercial en el país.

- Por medio de los valores obtenidos de retención y penetración, no se corroboró la facilidad relativa de las especies estudiadas citadas en la literatura.

- Se obtuvieron las curvas de retención y penetración, tanto lateral como transversal, en función del tiempo para cada una de las 
nueve especies maderables estudiadas, para cada tratamiento aplicado.

- A partir de la información obtenida, se determinaron los valores de percentil 50 de retención y penetración para cada tratamiento, así como el tiempo necesario para alcanzar dichos valores.

- Se obtuvieron valores observados de tiempo para la preservación de las especies estudiadas para cada tipo de preservante, tomando en cuenta los valores percentil 50 .

- Los valores obtenidos para el tiempo únicamente reflejan la cantidad necesaria de tiempo para alcanzar los resultados obtenidos de retención y penetración para cada especie en muestras de tamaño laboratorio; por lo que, para niveles industriales se deben realizar cambios de escala.

\section{RECOMENDACIONES}

Es recomendable realizar futuros estudios para determinar la efectividad de estos tratamientos ante los ataques de agentes biológicos, como son los hongos de pudrición, insectos, así como barrenadores marinos, dependiendo del uso final que vaya a tener cada especie maderable. Además, es recomendable realizar experimentos a una escala mayor antes de poder aplicar estos resultados a nivel industrial.

\section{AGRADECIMIENTOS}

Este proyecto fue financiado con ayuda de la Vicerrectoría de Investigación de la Universidad de Costa Rica a través del proyecto número 731-B2-022. Además, se agradece la ayuda y colaboración del técnico Pedro Rojas, así como de las asistentes de investigación Beatriz Alfaro, Tatiana Pardo y Mariana Vega.

\section{REFERENCIAS BIBLIOGRÁFICAS}

Alfaro, J. (2013). Medición de la penetración de tres tipos de preservantes en la madera de acuerdo al tiempo de tratamiento. Informe final de proyecto de investigación. San José, CR. Laboratorio de Productos Forestales, Universidad de Costa Rica.

AWPA (American Wood Protection Association). (2012). Method U1-12. User specification for treated wood. Maryland: US.

AWPA (American Wood Preservers' Association). (2011). Method A3-08. Standard methods for determining penetration of preservatives and fire retardants. Maryland: US.

AWPA (American Wood Preservers' Association). (1991). Method A3-91. Standard methods for determining penetration of preservatives and fire retardants. Maryland: US.

Berrocal, A; Muñoz, F; Gonzalez, G. (2004). Ensayo de penetrabilidad de dos preservantes a base de boro en madera de melina (Gmelina arborea) crecida en Costa Rica. Kurú: Revista Forestal 1(3): 1-12.

Blanco, L.; Carpio, I; Govaere, G. (2008). Análisis de teca (Tectona grandis L. F.) para dos edades y tres procedencias: Costa Rica, Tanzania y Tailandia, plantadas en el Bosque Tropical Seco del Pacífico Norte de Costa Rica. Informe final de proyecto de investigación. San José, CR. Laboratorio de Productos Forestales, Universidad de Costa Rica.

Blanco, L; Cruz, L. (1999). Evaluación de la resistencia a la degradación por insectos de algunas especies maderables de uso comercial bajo diferentes tratamientos. Informe final de proyecto de investigación 731-95-251. San José, CR. Laboratorio de Productos Forestales, Universidad de Costa Rica.

Carpio, I. (1992). Maderas de Costa Rica. 150 Especies forestales. San José: Editorial de la Universidad de Costa Rica.

Flores, E. (1999). La planta: estructura y función. Cartago: Libro Universitario Regional. Vol 1. 
Humar, M; Lesar, B. (2008). Fungicidal properties of individual components of copper-ethanolamine-based wood preservatives. International Biodeterioration \& Biodegradation 62(1): 46-50.

Ibach, R. (2005). Biological properties. En Rowell, R. (2005). Handbook of Wood Chemistry and Wood Composites. Boca Raton: CRC Press.

IPT (Instituto de Pesquisas Tecnológicas do Estado de São Paulo) (2001). Biodeterioração de madeiras em edificacões. São Paulo: IPT.

Lepage, E. et al (1986). Manual de preservação de madeiras. São Paulo: IPT. Vol 2.

Mora, N; Encinas, O. (2006). Biodegradación de maderas. Caracas, VE, Laboratorio Nacional de Productos Forestales.

Moya, R; Leandro, L; Monge, F. (2004). Preservación de melina mediante el método de inmersión-difusión con sales de boro. Kurú: Revista Forestal 1(2):1-4.

Purslow, D. (1976). The development of the double-vacuum process for preserving building timbers. Buckinghamshire, Building Research Establishment, Princes Risborough Laboratory (BRE Information, IS 20/76).

Yildiz, U; Temiz, A; Derya, E; Yildiz, S. (2004). Effects of the wood preservatives on mechanical properties of yellow pine (Pinus sylvestris L.) wood. Building and Environment 39(9):1071-1075.

Zabel, R; Morrell, J. (1992). Wood microbiology. Decay and its prevention. San Diego, US, Academic Press, Inc.

Zelinka, S; Sichel, R; Stone, R. (2010). Exposure testing of fasteners in preservative treated wood Gravimetric corrosion rates and corrosion product analyses. Corrosion Science 52(12): 3943-3948.

\section{SOBRE EL AUTOR}

Johnny Alfaro Pérez. Licenciado en Ingeniería Química. Docente de la Escuela de Ingeniería Quimica e Investigador del Instituto de Investigaciones en Ingeniería, Universidad de Costa Rica.

Correo electrónico: alfaro@uchicago.edu 
